\title{
LAMELLAR AND FIBRE BUNDLE MECHANICS OF THE ANNULUS FIBROSUS IN BOVINE INTERVERTEBRAL DISC
}

\author{
Claudio Vergari*, Jessica Mansfield, Judith R. Meakin, Peter C. Winlove \\ School of Physics and Astronomy, University of Exeter, Exeter, UK
}

\begin{abstract}
The intervertebral disc is a multicomposite structure, with an outer fibrous ring, the annulus fibrosus, retaining a gel-like core, the nucleus pulposus. The disc presents complex mechanical behaviour, and it is of high importance for spine biomechanics. Advances in multiscale modelling and disc repair raised a need for new quantitative data on the finest details of annulus fibrosus mechanics. In this work we explored inter-lamella and inter-bundle behaviour of the outer annulus using micromechanical testing and second harmonic generation microscopy. Twenty-one intervertebral discs were dissected from cow tails; the nucleus and inner annulus were excised to leave a ring of outer annulus, which was tested in circumferential loading while imaging the tissue's collagen fibres network with sub-micron resolution. Custom software was developed to determine local tissue strains through image analysis. Inter-bundle linear and shear strains were 5.5 and 2.8 times higher than intra-bundle strains. Bundles tended to remain parallel while rotating under loading, with large slipping between them. Inter-lamella linear strain was almost 3 times the intra-lamella one, but no slipping was observed at the junction between lamellae. This study confirms that outer annulus straining is mainly due to bundles slipping and rotating. Further development of disc multiscale modelling and repair techniques should take into account this modular behaviour of the lamella, rather than considering it as a homogeneous fibre-reinforced matrix.
\end{abstract}

Keywords: biomechanics; intervertebral disc; micromechanics; second harmonic generation 


\section{Introduction}

The intervertebral disc is the key element of spine flexibility; it resists high and diverse mechanical loadings while undergoing large and repeated strains. Disc mechanical behaviour is strongly dependent on its structure: the outer portion, the annulus fibrosus, is a strong ring of fibrous tissue that retains an inner core, the nucleus pulposus, which is gel-like in young, healthy animals and humans. The annulus is itself a composite structure formed of several lamellae, concentric layers containing fibres that are organised into bundles [1] and which are aligned within a lamella and at an angle between adjacent lamellae. When the spinal functional unit is subjected to physiological loading, annulus fibres can undergo high strains (up to $12 \%$ ) without apparent damage [2-4].

The micromechanical behaviour of the disc is of particular interest to better understand the aetiology and progression of disc disorders [5]. For instance, disc damage such as herniation and rupture often initiates with delamination and/or the propagation of small cracks [6]. This is driving disc modelling towards multiscale approaches [7], using sophisticated methods to incorporate fine details such as the interaction between fibre bundles. This, in turn, has raised the need for experimental micromechanical data on annulus ultrastructure. Such data are also required to inform the development of techniques of disc repair that aim at integrating a scaffold structure into the tissue [8,9]; indeed, cell survival and restoration of tissue strength depend on the mechanical compatibility between the engineered scaffold and the living tissue at the microscopic scale. A better knowledge of the latter could potentially help improve the design of these scaffolds.

The micromechanics of the whole disc have been investigated in some detail and a number of studies have focused on the mechanical properties of the annulus in vitro. For instance, uni- and bi-axial tests have been used to determine the stressstrain response of the annulus [10, 11], down to the level of single lamella $[12,13]$. Most previous work has, however, inferred the mechanical behaviour of the tissue by tracking cells $[14,15]$ or markers that were glued or photobleached on the tissue [10, 14-16], or alternatively relied on relatively low resolution tissue tracking (for instance, $0.2 \mathrm{~mm}^{2}$ elements $\left.[16,17]\right)$.

Second harmonic generation (SHG) is a multiphoton microscopic imaging technique that allows visualization of collagen network. It has been applied to investigate, for instance, cartilage [18, 19], cornea [20], tendon and ligament [21, 22] structure and mechanics, as well as structural disorder in intervertebral disc [23].

The aim of the present work was to describe and quantify the structural responses of the annulus subjected to micromechanical testing by means of SHG imaging, in order, particularly, to provide an insight on the mechanism of inter-bundle and inter-lamella straining.

\section{Materials and methods}

\subsection{Sample preparation}

Twenty-one intervertebral discs from cow tails were obtained from a local abattoir. Tails were frozen at $-20^{\circ} \mathrm{C}$ on the day of death and thawed overnight before testing (maximal frozen time: 5 months). Functional units were carefully dissected in order to expose the outer annulus fibrosus. The disc was detached from both adjoining endplates and its nucleus and inner annulus were excised to leave a ring of outer annulus of approximately $2 \mathrm{~mm}$ radial thickness (Fig.a). 


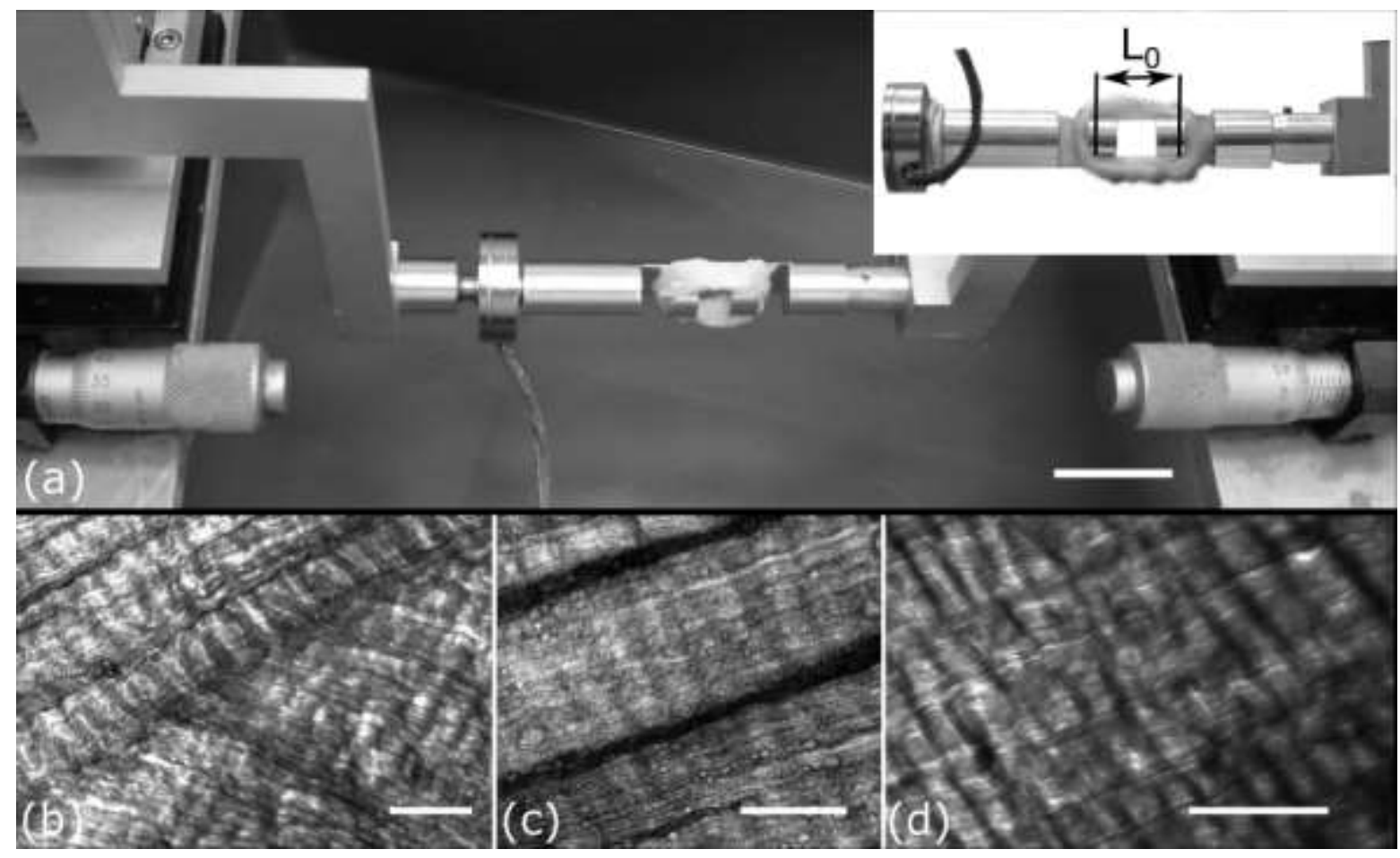

Fig.1. (a) Micromechanical testing rig (frontal view of the setup in the inset) and examples from the three sample groups: (b) lamellae with different orientations (Group L), (c) bundles with aligned fibres (Group B) and (d) homogeneous sample with no clear separation between bundles (Group F). Scale bars represent $2 \mathrm{~cm}$ in (a) and $100 \mu \mathrm{m}$ in (b-d). $\mathrm{L}_{0}$ is initial sample length, calculated between the inner faces of the flat hooks.

\subsection{Tensile testing}

A custom built micro-straining rig adapted from [24] was utilized to test the samples (Fig.a). The rig consists of two micromanipulation stages, each carrying a flat hook $8 \mathrm{~mm}$ in height. The sample was mounted on the hooks and the stages were used to move the hooks apart, thus applying circumferential strain to the outer annulus. The horizontal macroscopic strain $(\varepsilon)$ of the sample was measured from the displacement of the two stages, with 0.05 $\mathrm{mm}$ precision, as engineering strain: $\varepsilon=$ $\varepsilon_{H M}^{e n g}=100 *\left(L-L_{0}\right) / L_{0}$, where $\mathrm{L}_{\text {and }} \mathrm{L}_{0}$ are the initial and instantaneous distance between the inner faces of the flat hooks (Fig. 1), respectively. A load cell (LCM201 $100 \mathrm{~N}$, Omega, Manchester, UK) was placed in series with one of the hooks.
First, a preload of $0.2 \mathrm{~N}$ was applied and strain was zeroed. Then the sample was strained in $1 \%$ strain steps until the region of interest (ROI) could not be imaged anymore (see below). The sample was kept moist during the test by surface application of phosphate-buffered saline.

\subsection{Multiphoton Imaging}

The mechanical testing was performed under a confocal microscope (FluoView 300 and Olympus BX51) fitted with a 10x/0.4NA air objective (Olympus UPlanS Apo). The sample was illuminated with an 810-nm mode-locked femto-second Ti:Sapphire laser (Mira 900-D, Coherent Inc.) with a repetition rate of $76 \mathrm{MHz}$ and a pulse width of $100 \mathrm{fs}$ pumped by a $532 \mathrm{~nm}$ solid-state laser (Verdi V10, Coherent Inc.). This excites SHG in the sample, thus allowing visualization of collagen fibres 
(Fig.b-d.). SHG was collected in the backscattered direction using a photomultiplier (R3896 Hamamatsu) and the following combination of dichroic mirrors and filters to separate out the laser fundamental and any fluorescent signal; $670 \mathrm{~nm}$ long pass dichoric mirror (670dcxr Chroma), blue colour glass filter (CG-BG-39), narrow band pass filter (FF01-405/10 Semrock).

Samples were classed in three groups according to their apparent structure: Group L (Fig.b), for those ROIs where the fibres formed distinct lamellae, Group B (Fig.c), when the fibres were all parallel but formed bundles separated by a space in which no SHG was generated, and Group F (Fig.d), where the ROI showed a uniform array of fibres. In practice, group L ("lamellae") represents those ROIs where the intersection between two lamellae was visible. In group B ("bundles"), only one lamella was visible, with aligned but clearly delimited and separated bundles of fibres. Group F ("fibres") is similar to group B but the fibres were evenly distributes across the ROI, so that different bundles could not be clearly delimitated.

Images were acquired at each step of the mechanical test; the acquisition of an $800 \times 600$ pixels image (with sub-micron resolution) lasted about 30 seconds. When the ROI rotated out of the imaging plane and could not be imaged anymore, the test stopped.

\subsection{Image processing and strain calculation}

The centering of the ROI in the picture was improved after the test using ImageJ's plugin for linear alignment (translation only) with scale invariant features transform [25]; this rigid translation does not affect the strain field.

Custom software was written in Matlab 2014b (The MathWorks, Inc., Natick MA) to obtain a displacement map from each series of images and calculate instantaneous microscopic strains. The first image of the series was divided into square elements which were automatically tracked in the following images by digital image correlation (Supplementary Content 1). The tracking of an element was considered unreliable if the correlation was lower than 0.5 (this values was chosen from preliminary tests); the size of these elements was set as small as possible to optimize reliability for each series of images (resulting between 12.8 and $28.8 \mu \mathrm{m}^{2}$ ). If the correlation was unreliable in more than $2 \%$ of the elements after adapting the element size, a 2D Wiener adaptive noiseremoval filter was applied and the tracking run again. If the correlation was still unreliable in more than $2 \%$ of the elements, the image contrast was enhanced through histogram equalization. Finally, the remaining elements where the correlation failed (now less than 2\%) were tracked by minimization of the squared difference between the $n^{\text {th }}$ and $n^{\text {th }}-1$ element grayscale values, thus obtaining correct tracking of all elements. The displacement map was obtained at each frame from the element's displacement.

Displacements $\left(u_{x}\right.$ and $u_{y}$ for the horizontal and vertical directions, respectively) were filtered with a local quadratic regression to reduce noise (span parameter $=0.5$ ) and obtain smooth derivates to calculate local microscopic true strains. True linear $\left(\varepsilon_{\mathrm{x}}\right.$ and $\left.\varepsilon_{\mathrm{y}}\right)$ and shear $\left(\tau_{\mathrm{xy}}\right)$ strain maps were calculated from the displacements at each frame in a coordinate system fixed with the image frame, i.e., $\mathrm{x}$ was aligned with the transverse plane and $y$ with the vertical axis of the disc, as follows:

$$
\begin{aligned}
& \varepsilon_{x}=\ln \left(1+\partial u_{x} / \partial x\right) \\
& \varepsilon_{y}=\ln \left(1+\partial u_{y} / \partial y\right) \\
& \tau_{x y}=\ln \left(1+\partial u_{x} / \partial y\right)+\ln \left(1+\partial u_{y} / \partial x\right)
\end{aligned}
$$



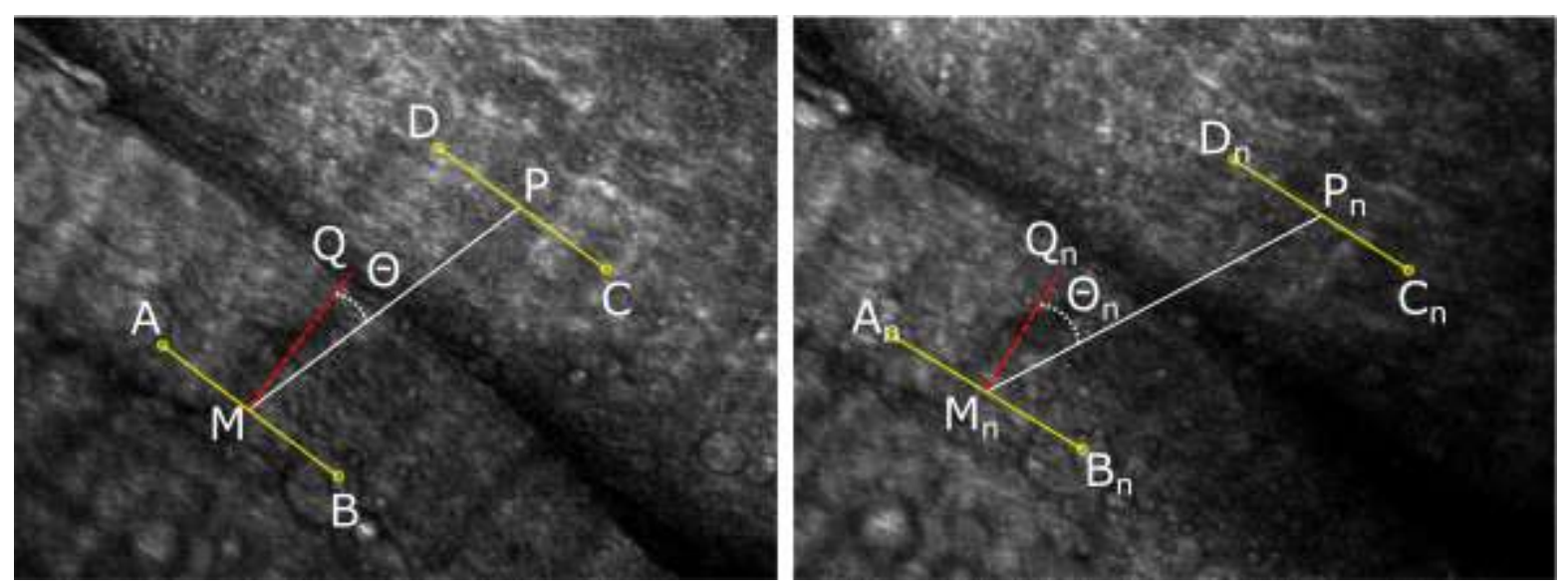

Fig. 2. Schematic representation of intra-bundle and inter-bundle strain calculation between the tissue at rest (left) and at 5\% macroscopic strain (right, $\mathrm{n}^{\text {th }}$ frame) in the horizontal direction (circumferential strain). Intra-bundle linear strain is calculated as the average strain of the $\mathrm{AB}$ and $\mathrm{CD}$ segments. Intra-bundle shear is the average shear in the neighbourhood of the points $\mathrm{A}, \mathrm{B}, \mathrm{C}$ and $\mathrm{D}$, calculated in the fibres direction. Inter-bundle strain is the strain of the MP segment projected on the MQ segment, while inter-bundle shear is the summed variation of the AMP and CPM angles.

The tracking technique and calculations were validated by measuring strains in a series of images that were artificially strained using GIMP 2.8 (www.gimp.org). The sensitivity to the tracking element size was also assessed.

\subsection{Intra- and inter-bundle strain calculations}

In addition to local strain maps, four specific strain definitions were introduced to estimate the kinematics of bundles and lamellae: intra- and inter-bundle linear strain, and intra- and inter-bundle shear strain. Intra- and inter-lamella strains (calculated in group L) have the same definition of bundle strains (group B), the main difference being that the segments in group $\mathrm{L}$ are at an angle while in group B they are almost parallel. Moreover, the intra-bundle and intra-lamella strains had the same definition and interpretation, i.e. the change of fibres length, so they were pooled to calculate overall averages.

In order to calculate these strains, a segment was defined in each lamella or fibre bundle (Fig. 2, segments $\mathrm{AB}$ and $\mathrm{CD}$ ), aligned with the fibres, in the first image of the series (i.e., at rest). The endings of each segments were linked to the nine closest tracking elements and their position recalculated at each frame to follow the tissue deformation.

Intra-bundle linear strain at the $\mathrm{n}^{\text {th }}$ frame was defined as the average true strain of the $\mathrm{AB}\left(\varepsilon_{/ /}^{A B}\right)$ and $\mathrm{CD}\left(\varepsilon_{/ /}^{C D}\right)$ segments:

$\varepsilon_{/ /}=\frac{\left|\varepsilon_{/ /}^{A B}\right|+\left|\varepsilon_{/ /}^{C D}\right|}{2}=0.5 \cdot\left(\left|\log \frac{\overline{A_{n} B_{n}}}{\overline{A B}}\right|+\left|\log \frac{\overline{C_{n} D_{n}}}{\overline{C D}}\right|\right)$

This strain represents the change in length of the bundles (or of the lamellae) in the direction of the fibres. Absolute values were calculated to compare strain magnitude regardless whether the fibres were in tension or compression. Interbundle linear strain $\left(\varepsilon_{\perp}\right)$ was defined as the true strain of MP projected on MQ, which is orthogonal to $\mathrm{AB}$ :

$$
\varepsilon_{\perp}=\varepsilon_{\perp}^{M P}=\left|\log \frac{\overline{M_{n} P_{n}} \cos \theta_{n}}{\overline{M P} \cos \theta}\right|
$$




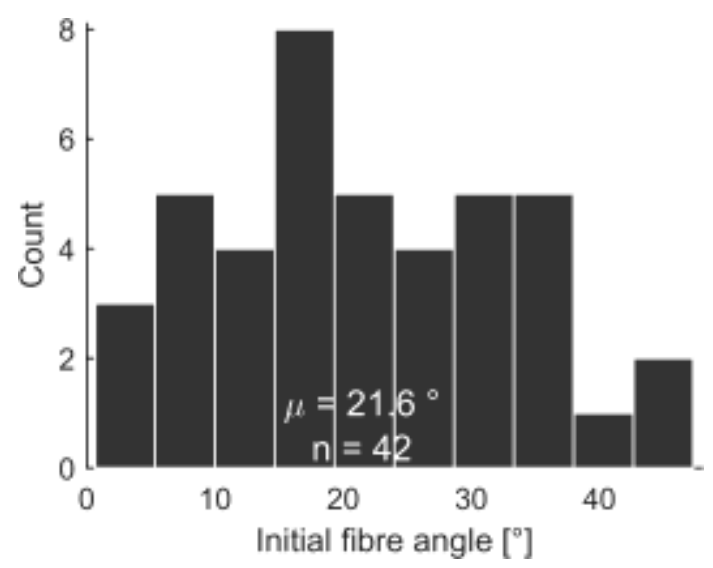

Fig. 3. Distribution of initial orientation of fibres, relative to the horizontal (which corresponds to the intervertebral disc transversal plane). The total count is higher than the number of samples because each lamella and each bundle was counted separately.

This strain reflects how bundles or lamellae move closer or apart to each other. The projection of MP was used instead of MP in order to avoid an apparent increase of inter-bundle linear strain when the bundles slide against each other. Such movement would lengthen (or shorten) the MP segment but not its projection on MQ.

Intra-bundle shear strain was calculated as the average absolute shear calculated in the fibre direction in the previously described strain map, and measured in the 9 tracking elements closer to each end of the $\mathrm{AB}$ and $\mathrm{CD}$ segments. This represents the shear strain in the core of the bundle (or lamella). Inter-bundle shear was calculated as the sum of the absolute angular variations between bundles:

$$
\begin{aligned}
\tau_{\perp}=\left|\tau_{\perp}^{A M P}\right|+ & \left|\tau_{\perp}^{C P M}\right| \\
& =\left|\mathrm{A}_{n} \widehat{\mathrm{M}_{n} \mathrm{P}_{n}}-\widehat{\mathrm{AMP}}\right| \\
& +\left|\mathrm{C}_{n} \widehat{\mathrm{P}}_{n} \mathrm{M}_{n}-\widehat{\mathrm{CPM}}\right|
\end{aligned}
$$

This shear strain represents the change in angle between bundles (or lamellae). Angles changes were considered as absolute values so that any possible interbundle kinematic that could affect those angles would add up to a positive value; in practice, the direction of the shearing is lost but the amounts of shearing are accounted for.

These strains were calculated from the change in length of segments "anchored" to the fibre bundles (or lamellae), and from changes in their relative angles. Therefore, they are independent of the coordinate system.

\subsection{Statistics}

Correlations between fibre angles and strain were analysed with Spearman's rank correlation while differences between average strains were analysed with Wilcoxon rank sum test to account for nonnormal data. A Kruskal-Wallis test was used to analyse strain differences between groups $\mathrm{B}, \mathrm{L}$ and $\mathrm{F}$; significance was set at 0.05 . Linearity of relationships was analysed by fitting linear approximations to the data and measuring the root mean squared error (RMSE) between the fit and the data.

\section{Results}

The final macroscopic strain achieved over all specimens was $16.1 \pm 8.9 \%$ (ranging between 5 and $32 \%$ ). No macroscopic or microscopic failures (i.e., sudden changes in strain or visible tissue ruptures) were observed in any of the specimens even at higher strains; however, in three samples the horizontal microscopic strain decreased sharply before the macroscopic strain reached $5 \%$, which was taken to indicate failure on a microscopic scale outside the imaging region, and so they were removed from the following analysis.

\subsection{Fibres orientation}

Fibres had an average inclination at rest of $21.6^{\circ} \pm 11.9^{\circ}$ relative to the disc transverse plane (i.e., the horizontal in 
Fig.b), ranging from $0.6^{\circ}$ to $47^{\circ}$ (Fig.). Fibres angles varied linearly with the applied macroscopic strain (Fig. 2), with significant re-orientation towards the direction of the applied strain (Fig. 1, p < 0.001 between the angles at rest and at $5 \%$ strain). Angle variation with macroscopic strain varied linearly within an average RMSE of $0.26^{\circ}$ (ranging between $0.05^{\circ}$ and $1.2^{\circ}$ ).

\subsection{Microscopic strain}

The annulus ROIs showed three structural patterns, as illustrated in Fig.1. Six samples were classed in Group L, nine samples in Group B and three samples in Group $\mathrm{F}$ according to the imaged ROI. These features depended on the position of the ROI; regions belonging to different groups could be observed in the same sample.

Despite the structural differences, macroscopic and microscopic horizontal strains varied linearly (Fig. 3), with an average RMSE of $0.15 \%$ strain (ranging between $0.05 \%$ and $0.4 \%$ ) between the data and their linear approximation. The slopes of these linear approximations did not vary between groups $(\mathrm{H}=4.31, \mathrm{p}=0.12$,

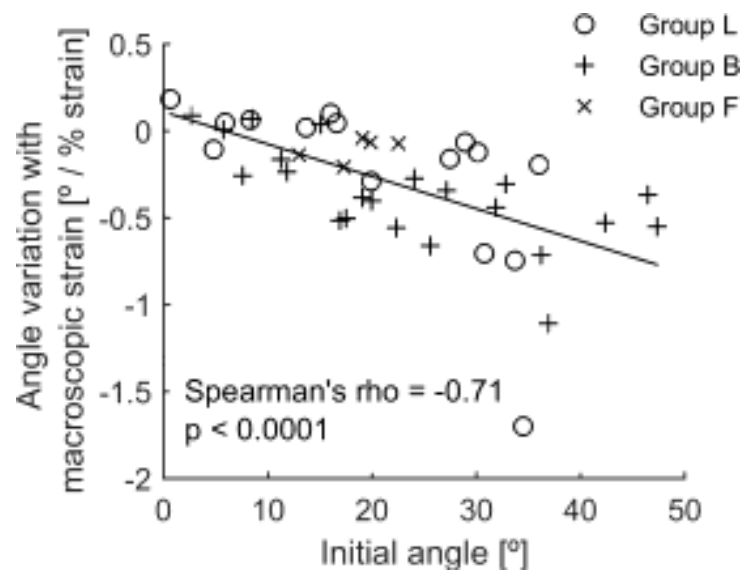

Fig. 1. Relation between the fibre angle at rest and the angle variation with macroscopic strain; the relation was statistically significant (regression line: $\mathrm{y}$ $=-0.019 \mathrm{x}+0.11)$.

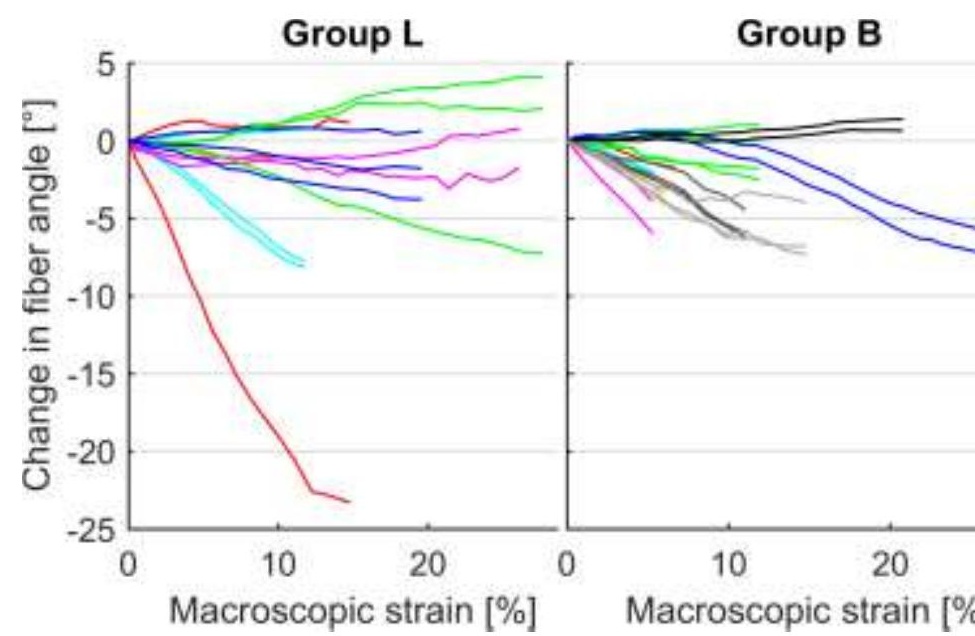

Fig. 2. Fibre angle variation with macroscopic strain in $\mathrm{g}$ $\mathrm{F}$ (homogeneous fibres). The definition of the three grou the same colour in each group represent different lamella the same sample.

Kruskal-Wallis test) and the average slope was $0.33 \pm 0.43$; i.e., microscopic horizontal strain increased by about a third of the applied macroscopic strain. A change of slope can be seen in several of the graphs, indicating that at some point during tension the rate of microscopic strain started decreasing.

Microscopic vertical strains were negative but much higher (Fig. 3), and had a linear variation with macroscopic horizontal strain (RMSE $=0.18 \%$ strain). The average slope was $-1.1 \pm 0.8$, indicating that a macroscopic strain applied in a horizontal direction was associated with a similar vertical strain. No difference was observed between groups $(\mathrm{H}=2.2, \mathrm{p}=$ $0.33)$.

\subsection{Inter-lamella and inter-bundle strains}

Intra- and inter-lamella strain is shown in Fig. 4 against macroscopic strain, and compared to intra- and inter-bundle strains. These strains were calculated at $5 \%$ macroscopic strain in order to compare all samples under the same conditions. Indeed, the tests were stopped when the ROI rotated out of the imaging plane; for some samples 


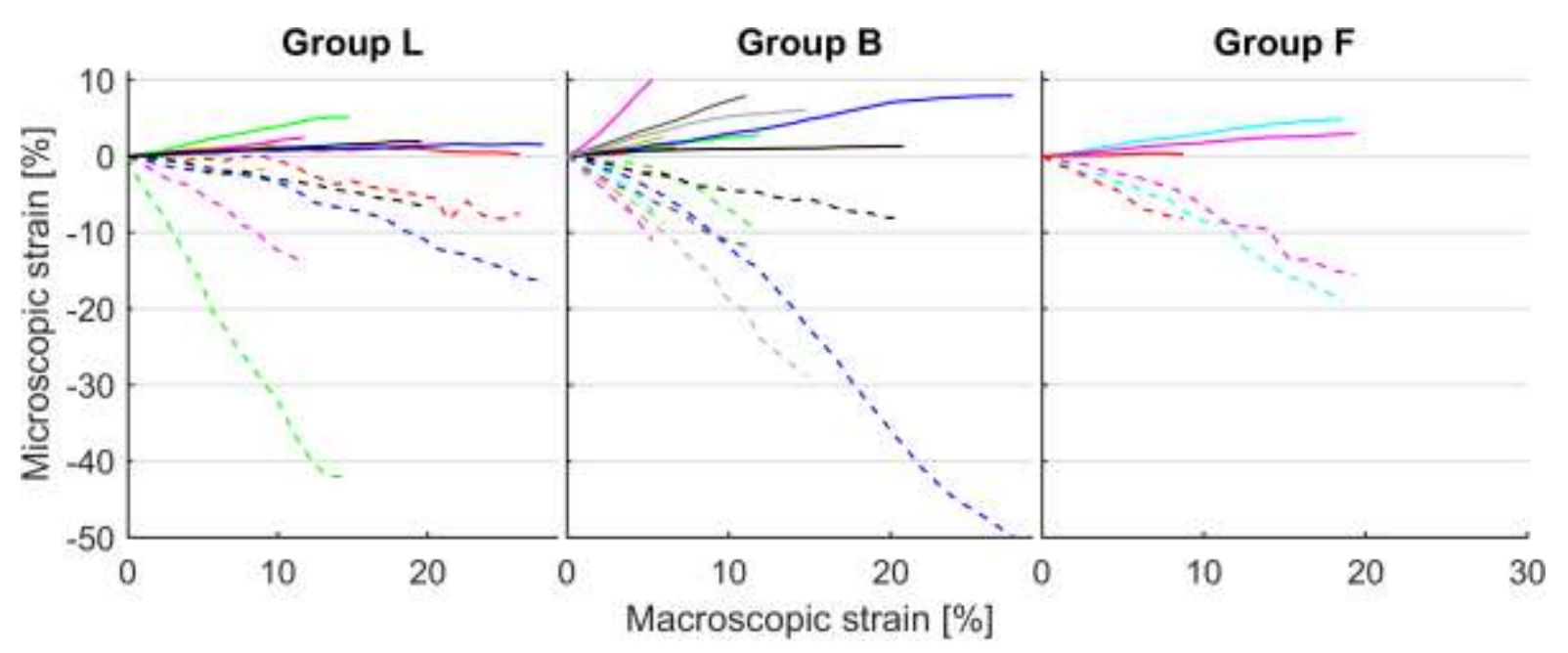

Fig. 3. Relationship between microscopic and macroscopic strain in the horizontal (i.e., the loading direction, solid lines) and vertical direction (dashed lines) in group L (lamellae), B (bundles) and F (homogeneous fibres). Same colour indicates same sample. Each curve could be approximated by a line with root mean squared errors between 0.05 and $0.4 \%$ strain. A decrease in microscopic strain rate can be observed in some samples, but it did not correspond to significant changes in the samples (i.e., no clear ruptures were observed). Colours reflect samples in Fig. 2.

it happened at $5 \%$ macroscopic strain. Intra-lamella and intra-bundle strain values were pooled because they represent the same strain in the bulk of the tissue and their difference was not significant (Wilcoxon test, $\mathrm{U}=22, \mathrm{p}=0.58$ ); average linear strain was $1.2 \pm 0.8 \%$ while shear strain was 2.1 $\pm 1.8^{\circ}$.

Samples in Group L (Fig.b, Additional Content 1) showed inter-lamella linear and shear strain of $3.4 \pm 5.2 \%$ and $2.0 \pm 2.0^{\circ}$, respectively. Samples in Group B (Fig.c, Additional Content 2) showed inter-bundle linear and shear strain of $6.6 \pm 6.7 \%$ and $5.9 \pm 6.0^{\circ}$, respectively. Samples in Group $\mathrm{F}$ appeared homogeneous so inter-bundle strain was not evaluated. Strain in the bulk of the tissue (intra-lamella and intra-bundle) was significantly lower than inter-bundle and inter-lamella strain (Wilcoxon test, $\mathrm{U}=$ $51, \mathrm{p}=0.01)$.

\subsection{Typical examples}

Additional Content 1 shows an example of the behaviour of Group L; the fibres of the top-right lamella have an initial angle of $8^{\circ}$ with the horizontal (i.e., the direction of loading) which slightly increases to $9^{\circ}$ during loading. This lamella strains by $6 \%$ in the fibre direction. The orientation of the bottom-left lamella goes from $34^{\circ}$ to $26^{\circ}$, while its length decreases by $-2 \%$ in the fibre direction. The horizontal strain, aligned to the loading, is concentrated in the lamella appearing at the top of the image, while the lamella appearing at the bottom tended to shorten in this direction as well. In general, lamellae with small angles had a tendency to strain while those with larger angles tended to rotate and align with the strain, with small or even negative linear strains; confirming this, a negative correlation was observed between the magnitude of fibre angle at rest and intralamella strain at $5 \%$ macroscopic strain (Spearman's rho $=-0.71, p=0.004$ ). The same correlation was significant in the Group B (Spearman's rho $=-0.52, p=0.02$ ).

No slipping or strain concentrations are visible at the junction between lamellae, neither in this sample nor in the other samples belonging to this group. 


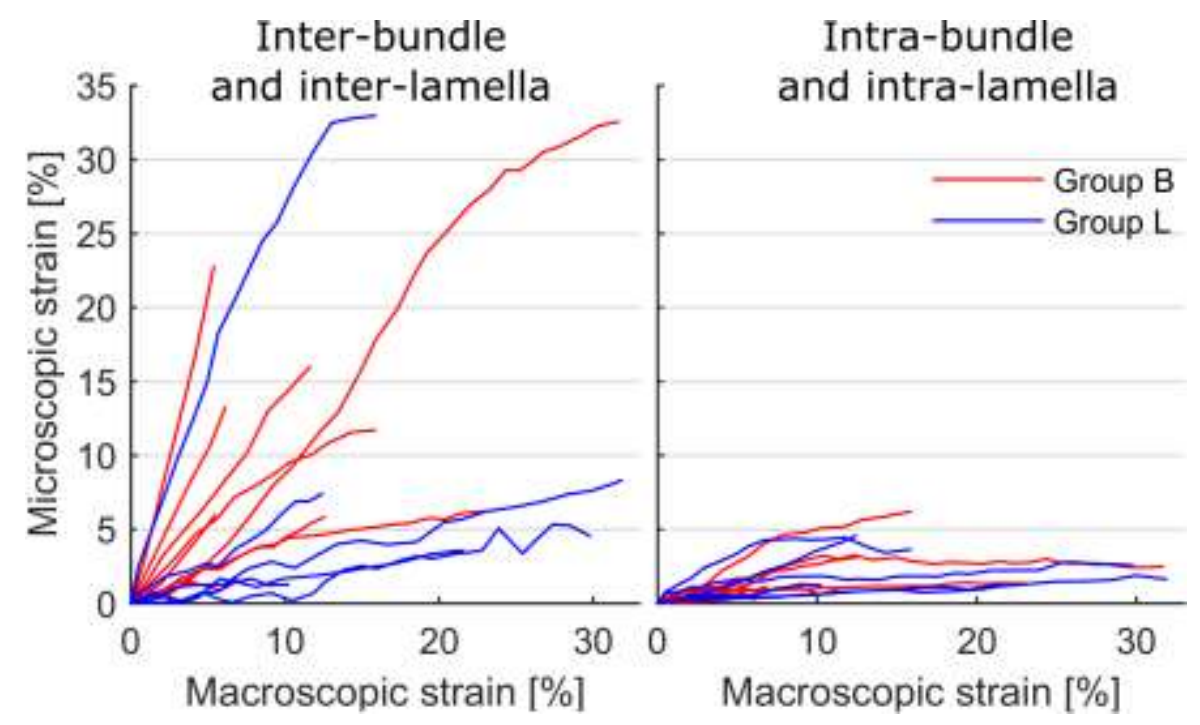

Fig. 4. Inter-bundle and inter-lamella strain (left) against macroscopic strain in the horizontal direction (i.e., the loading direction), compared to intra-bundle and intra-lamella strain (right). A difference can also be discerned between group B ("bundles") and group L ("lamellae").

Additional Content 2 illustrates the behaviour of Group B; the animation shows horizontal straining between rest and $5 \%$ macroscopic strain. The slipping between bundles is very apparent, as is the stress concentration in the inter-bundle space. The vertical strain in the inter-bundle space is negative, as is evident from the grid representation where both bundles move vertically towards each other.

The fibres angles of the two bundles rotate from $36^{\circ}$ and $37^{\circ}$ to $32^{\circ}$ and $31^{\circ}$, respectively, thus remaining practically parallel. Indeed, lamellae from the same sample had an average difference in orientation of $38 \pm 15^{\circ}$, while bundles from the same sample were parallel with an initial difference in orientation of $2 \pm 2^{\circ}$. The angle between lamellae changed by $3^{\circ}$ in average, at 5\% strain, while angles between bundles changed by $0.6^{\circ}$, confirming that parallel bundles tend to remain parallel while lamellae can vary their respective angle.

\section{Discussion}

The aim of the present work was to obtain information on the local strain field of the outer annulus, and in particular to quantify the inter-lamella and inter-bundle mechanical behaviour. Tissue behaviour was quantified with high resolution image tracking (between 12.8 and $28.8 \mu \mathrm{m}^{2}$ ), and the interaction between lamellae and fibre bundles was investigated. The applied loading did not aim at reproducing a physiological loading of the disc, but at obtaining large homogeneous strains of the outer annulus.

One limitation of the present study is the use of young animal samples; aging and inter-species differences can affect disc micromechanics. However, the normal bovine disc is of interest in its own right since it is often used as a model for human discs because of its size and mechanical behaviour [14, 26, 27]. A second limitation is that the viscoelastic behaviour of the tissue was neglected. The delay between a strain step and image acquisition was relatively small (50 seconds in average); it 
was not possible to wait for the tissue to stabilize at each step, because preliminary measurements showed that load relaxation continued for at least 1 hour after a strain step.

Samples did not fail even with macroscopic strains of up to $28 \%$. This is consistent with previous studies where human annulus samples failed at $65 \%$ strain [28]. Another study reports smaller failure values $(10-20 \%$, [29]), but the samples tested in that work were cut in dumbbell shapes, thus probably altering the continuity of the fibres and therefore weakening the tissue.

The microscopic fibre strains $(1.2 \%$, ranging from 0.1 to $3.4 \%$ ) obtained at $5 \%$ macroscopic strain were actually smaller than the maximal strains observed in vitro in human functional units during different types of complex loading [2]. That the microscopic strain is smaller than the macroscopic applied strain is due to the inhomogeneous character of the annulus [14]; the macroscopic strains result from the summation of complex interactions between bundles and lamellae. However, the macroscopic strains in this work were directly measured from the displacement of the micromanipulation stages; the actual strain applied to the free part of the annulus ring was therefore overestimated, because of the compression of the portion of the tissue in contact with the vertical flat hooks (Fig.). Moreover, the outer annulus ring, i.e., the site of the microscopic imaging, was less strained than the inner portion because of the loading technique.

Orientation of fibres within lamellae was between $\sim 0^{\circ}$ and $47^{\circ}$ in magnitude. While lamellae with an almost horizontal orientations are not commonly described in the literature, the maximal and average values are consistent with previous works on human and ovine discs [13, 30]. Preparation of the sample aimed at leaving the annulus multi-layered structure as intact as possible, but it is possible that a decrease in fibre angle was due to the removal of the endplates, which allowed a rotation of the lamellae towards the disc mid-plane. The removal of the endplates also allowed large transverse shrinkage of the sample under loading. However, the free boundary condition given by the endplate removal allowed testing the annulus as a material, i.e., its behaviour independent of the structural constraints imposed on it as part of the spinal function unit.

Michalek et al. [31] previously reported that inter-lamella shearing strain was mainly due to skewing rather than sliding. This is corroborated by our results, since inter-lamella shear strain was similar to intra-lamella, and no sliding was observed between lamellae, thus confirming a strong inter-lamella cohesion [32, 33]. Indeed, it has been previously shown that interlamella shear resistance confers compressive stiffness to the disc [26].

Inter-lamella linear strain was almost 3 times higher than the intra-lamella one. This is due to two phenomena: the lamellae getting closer or further away from each other due to the changes in their fibre alignment, and the fibre bundles being pulled apart or closer to each other. Previous work by Mengoni et al. [34] reported an inter-lamella stiffness higher than intra-lamella. Those authors, however, applied a very specific loading to a thin radial slice of annulus whereas in this case the composite-like structure of the annulus was not compromised.

Inter-bundle linear and shear strain were much higher than intra-bundle strains (5.5 and 2.8 times higher, respectively); large slipping was observed between bundles, accounting for these strains. This was previously inferred from using cell displacement as a strain marker [14, 15], obtaining similar strain magnitudes, and led to the proposal that inter-bundle slipping is the main component of strain within a lamella. Bruehlmann et al., however, concluded that intra-lamella cells are 
relatively shielded from strains; our results suggest that cells in the inter-bundle space could actually be subjected to high shear.

Bundles within a lamella tended to remain parallel whilst sliding and rotating (Additional Content 2). This can explain the negative inter-cellular strains previously observed [14]: sliding between bundles can actually push those cells in the inter-bundle space cells closer to each other. The sliding also allows the reorientation of the lamella with relatively small intra-bundle strain. This is consistent with the threedimensional inter-bundle connectivity described by Pezowicz et al. [32] and Yu et al. [1]; it seems that the mechanical role of the elastic inter-bundle connection is to allow them this mobility. Such mobility could be reduced with the increase in number and stiffness of elastin crossbridges and bundles interconnections which occurs with aging $[33,35]$.

\section{Conclusion}

This work investigated the complex microscopic mechanical behaviour of the outer annulus. The resulting data can be used to develop and validate multiscale models of the intervertebral disc, and to develop better adapted scaffolds for annulus repair. In particular, our results confirm that lamellae, which are often modelled as a fibre-reinforced homogeneous matrix, are actually a complex composite formed up by bundles of fibres that slide relative to each other. This important result suggests that the attention should shift from inter-lamella mechanics to inter-bundle mechanics. It is therefore an immediate challenge to develop multiscale model able to accurately reproduce inter-lamella behaviour by implementing consistent inter-bundle linking.

\section{Acknowledgements}

The authors are grateful to the Henry Smith Charity for funding.

\section{Disclosures}

No conflict of interest to disclose.

\section{References}

[1] Yu J, Schollum ML, Wade KR, Broom ND, Urban JPG, ISSLS Prize Winner: A Detailed Examination of the Elastic Network Leads to a New Understanding of Annulus Fibrosus Organization, Spine. 40 (2015) 1149-57.

[2] Heuer F, Schmidt H, Wilke H-J, The relation between intervertebral disc bulging and annular fiber associated strains for simple and complex loading, J Biomech. 41 (2008) 1086-94.

[3] Cannella M, Arthur A, Allen S, Keane M, Joshi A, Vresilovic E, et al., The role of the nucleus pulposus in neutral zone human lumbar intervertebral disc mechanics, J Biomech. 41 (2008) 2104-11.

[4] Nachemson AL, The Influence of Spinal Movements on the Lumbar Intradiscal Pressure and on the Tensile Stresses in the Annulus Fibrosus, Acta Orthopaedica. 33 (1963) 183207. 
[5] Gregory DE, Bae WC, Sah RL, Masuda K, Disc degeneration reduces the delamination strength of the annulus fibrosus in the rabbit annular disc puncture model, The Spine Journal. 14 (2014) 1265-71.

[6] Iatridis JC, ap Gwynn I, Mechanisms for mechanical damage in the intervertebral disc annulus fibrosus, J Biomech. 37 (2004) 1165-75.

[7] Reutlinger C, Burki A, Brandejsky V, Ebert L, Buchler P, Specimen specific parameter identification of ovine lumbar intervertebral discs: On the influence of fibre-matrix and fibre-fibre shear interactions, J Mech Behav Biomed Mater. 30 (2014) 279-89.

[8] Long RG, Bürki A, Zysset P, Eglin D, Grijpma DW, Blanquer SBG, et al., Mechanical Restoration and Failure Analyses of a Hydrogel and Scaffold Composite Strategy for Annulus Fibrosus Repair, Acta Biomaterialia.

[9] Bron J, Helder M, Meisel H-J, Van Royen B, Smit T, Repair, regenerative and supportive therapies of the annulus fibrosus: achievements and challenges, Eur Spine J. 18 (2009) 30113.

[10] O'Connell GD, Sen S, Elliott DM, Human annulus fibrosus material properties from biaxial testing and constitutive modeling are altered with degeneration, Biomech Model Mechanobiol. 11 (2012) 493-503.

[11] Wagner DR, Lotz JC, Theoretical model and experimental results for the nonlinear elastic behavior of human annulus fibrosus, J Orthop Res. 22 (2004) 901-9.

[12] Monaco LA, DeWitte-Orr SJ, Gregory DE, A comparison between porcine, ovine, and bovine intervertebral disc anatomy and single lamella annulus fibrosus tensile properties, Journal of Morphology. (2015) n/a-n/a.

[13] Holzapfel GA, Schulze-Bauer CAJ, Feigl G, Regitnig P, Single lamellar mechanics of the human lumbar anulus fibrosus, Biomech Model Mechanobiol. 3 (2005) 125-40.

[14] Bruehlmann SB, Hulme PA, Duncan NA, In situ intercellular mechanics of the bovine outer annulus fibrosus subjected to biaxial strains, J Biomech. 37 (2004) 223-31.

[15] Bruehlmann SB, Matyas JR, Duncan NA, ISSLS prize winner: Collagen fibril sliding governs cell mechanics in the anulus fibrosus: an in situ confocal microscopy study of bovine discs, Spine. 29 (2004) 2612-20.

[16] Karakolis T, Callaghan JP, Localized strain measurements of the intervertebral disc annulus during biaxial tensile testing, Comput Methods Biomech Biomed Eng. 18 (2014) 1737-43.

[17] Baldit A, Ambard D, Cherblanc F, Royer P, Experimental analysis of the transverse mechanical behaviour of annulus fibrosus tissue, Biomech Model Mechanobiol. 13 (2014) $643-52$.

[18] Mansfield JC, Winlove CP, Moger J, Matcher SJ, Collagen fiber arrangement in normal and diseased cartilage studied by polarization sensitive nonlinear microscopy, J Biomed Opt. 13 (2008) 044020.

[19] Cox G, Kable E, Jones A, Fraser I, Manconi F, Gorrell MD, 3-Dimensional imaging of collagen using second harmonic generation, Journal of Structural Biology. 141 (2003) 5362 . 
[20] Thomasy SM, Raghunathan VK, Winkler M, Reilly CM, Sadeli AR, Russell P, et al., Elastic modulus and collagen organization of the rabbit cornea: Epithelium to endothelium, Acta Biomaterialia. 10 (2014) 785-91.

[21] Legerlotz K, Dorn J, Richter J, Rausch M, Leupin O, Age-dependent regulation of tendon crimp structure, cell length and gap width with strain, Acta Biomaterialia. 10 (2014) 444755.

[22] Henninger HB, Valdez WR, Scott SA, Weiss JA, Elastin governs the mechanical response of medial collateral ligament under shear and transverse tensile loading, Acta Biomaterialia. 25 (2015) 304-12.

[23] Reiser KM, Bratton C, Yankelevich DR, Knoesen A, Rocha-Mendoza I, Lotz J, Quantitative analysis of structural disorder in intervertebral disks using second harmonic generation imaging: comparison with morphometric analysis, J Biomed Opt. 12 (2007) 064019--17.

[24] Mansfield JC, Bell JS, Winlove CP, The micromechanics of the superficial zone of articular cartilage, Osteoarthr Cartilage. 23 (2015) 1806-16.

[25] Lowe D, Distinctive Image Features from Scale-Invariant Keypoints, International Journal of Computer Vision. 60 (2004) 91-110.

[26] Adam C, Rouch P, Skalli W, Inter-lamellar shear resistance confers compressive stiffness in the intervertebral disc: An image-based modelling study on the bovine caudal disc, $\mathbf{J}$ Biomech. (In Press).

[27] Michalek AJ, Buckley MR, Bonassar LJ, Cohen I, Iatridis JC, The effects of needle puncture injury on microscale shear strain in the intervertebral disc annulus fibrosus, The Spine Journal. 10 (2010) 1098-105.

[28] Green TP, Adams MA, Dolan P, Tensile properties of the annulus fibrosus II. Ultimate tensile strength and fatigue life, Eur Spine J. 2 (1993) 209-14.

[29] Ebara S, Iatridis JC, Setton LA, Foster RJ, Mow VC, Weidenbaum M, Tensile properties of nondegenerate human lumbar anulus fibrosus, Spine. 21 (1996) 452-61.

[30] Reid JE, Meakin JR, Robins SP, Skakle JMS, Hukins DWL, Sheep lumbar intervertebral discs as models for human discs, Clin Biomech. 17 (2002) 312-4.

[31] Michalek AJ, Buckley MR, Bonassar LJ, Cohen I, Iatridis JC, Measurement of local strains in intervertebral disc anulus fibrosus tissue under dynamic shear: contributions of matrix fiber orientation and elastin content, J Biomech. 42 (2009) 2279-85.

[32] Pezowicz CA, Robertson PA, Broom ND, The structural basis of interlamellar cohesion in the intervertebral disc wall, J Anat. 208 (2006) 317-30.

[33] Schollum ML, Robertson PA, Broom ND, How age influences unravelling morphology of annular lamellae - a study of interfibre cohesivity in the lumbar disc, J Anat. 216 (2010) $310-9$.

[34] Mengoni M, Luxmoore BJ, Wijayathunga VN, Jones AC, Broom ND, Wilcox RK, Derivation of inter-lamellar behaviour of the intervertebral disc annulus, J Mech Behav Biomed Mater. 48 (2015) 164-72.

[35] Yu J, Winlove PC, Roberts S, Urban JP, Elastic fibre organization in the intervertebral discs of the bovine tail, J Anat. 201 (2002) 465-75. 
Accepted Manuscript. Acta Biomaterialia.

The original publication is available at DOI: http://dx.doi.org/10.1016/j.actbio.2016.04.002 